\title{
Effects of complex decongestive therapy on quality of life, depression, neuropathic pain, and fatigue in women with breast cancer-related lymphedema
}

\author{
Hilal Yesil, ${ }^{1}$ Sibel Eyigör, ${ }^{2}$ İsmail Caramat, ${ }^{2}$ Rıdvan Işık ${ }^{2}$ \\ ${ }^{1}$ Department of Physical Medicine and Rehabilitation, Medical Faculty of Afyon Kocatepe University, Afyon, Turkey \\ ${ }^{2}$ Department of Physical Medicine and Rehabilitation, Medical Faculty of Ege University, İzmir, Turkey \\ Received: July 2016 Accepted: November 2016
}

\section{ABSTRACT}

Objectives: To investigate the effects of complex decongestive therapy (CDT) on the quality of life, depression, neuropathic pain, and fatigue in patients with breast cancer-related lymphedema (BCRL).

Patients and methods: Between March 2015 and June 2015, a total number of 60 patients (mean age $55.7 \pm 10.3$ years; range 18 to 85 years) with BCRL were included in the study. Demographic data and previous medical records were recruited from medical files. The European Organization for Research and Treatment of Cancer Quality of Life- C30 (EORTC QLQ-C30) for the quality of life, the Brief Fatigue Inventory (BFI) for fatigue, Douleur Neuropathique 4 Questions (DN4) for neuropathic pain, and the Beck Depression Inventory (BDI) for the emotional status were used before and after the treatment. All patients received 20 sessions (one hour) of CDT for four weeks (five days per week).

Results: There was a statistically significant reduction in the volume of the involved limbs after the treatment $(\mathrm{p}<0.001)$. There was also a significant reduction in the general health and functional scores of the EORTC QLQ-C30 ( $\mathrm{p}<0.001, \mathrm{p}=0.004$, respectively). The DN4, BFI, and BDI scores were significantly improved after the treatment $(\mathrm{p}<0.001, \mathrm{p}=0.043, \mathrm{p}=0.019$, respectively).

Conclusion: Our study results suggest that CDT is an effective and safe method to achieve not only a significant volume reduction in the limbs involved by lymphedema, but also good outcomes in the management of other symptoms related to BCRL.

Keywords: Complex decongestive therapy; lymphedema; quality of life.

Breast cancer is the most common type of malignancy in female population across the globe, and nearly 1.68 million new cases are diagnosed annually. ${ }^{[1]}$ Despite the significant increase in the survival rates, many problems associated with either the disease itself or its treatment still persists in patients living with the disease. ${ }^{[2]}$ Secondary lymphedema (LE), one of the well-known complications of breast cancer treatment, is defined as an accumulation of proteinrich interstitial fluid due to the insufficient capacity of the lymphatic system. ${ }^{[3,4]}$

Previous studies have demonstrated that those who suffer from LE usually experience a variety of problems, including feelings of discomfort and heaviness, loss of strength and function, psychological distress, depression, low self-esteem, fatigue, neuropathic pain in the affected arm, and an elevated risk of recurrent infections. ${ }^{[5-7]}$ These complications may cause significant deterioration in the quality of life (QoL). ${ }^{[8]}$ Besides, management of LE which, indeed, is a progressive and chronic complication, is associated with a high economic burden; therefore, it is of utmost importance to identify and manage these symptoms or complications in patients with breast cancer-related lymphedema (BCRL) ${ }^{[9]}$

Furthermore, LE has been known to be associated with numerous symptoms and complications; thus, it is critical to start the treatment protocol immediately 
after the diagnosis, irrespective of the severity or stage of the disease. Nonetheless, treatment of LE remains a major challenge for both patients and health care professionals. Recently, standard protocol for the management of LE includes complete decongestive therapy (CDT), which comprises the use of manual lymph drainage (MLD), daily bandaging, skin care, exercise, and compression. ${ }^{[10]}$ This procedure involves two stages: an intensive phase to reduce and an additional maintenance phase to stabilize LE volume. It should be noted that satisfying outcomes are highly dependent upon the patient compliance; however, it has been reported that the rate of favorable results varies between 22 to $73 \%{ }^{[11]}$

A comprehensive review of the literature reveals satisfying results of CDT in reducing the volume of LE. However, few studies have addressed into the problems associated with LE, such as QoL, depression, fatigue, and neuropathic pain. Therefore, in the present study, we aimed to investigate the effects of CDT on these parameters in patients with BCRL.

\section{PATIENTS AND METHODS}

Patients who were admitted to the outpatient clinic of Physical Medicine and Rehabilitation of our university hospital and diagnosed with BCRL were included in the study. Patients were referred to our outpatient clinic after complete medical evaluations in external first or second-degree medical centers, and other causes of extremity swelling such as tumor recurrence or metastases were ruled out. Patients aged between 18 and 85 years and having BCRL were included in the study. Exclusion criteria included acute inflammation, history of recurrent infections, ulcers in the involved extremity, significant congestive heart failure, and acute deep vein thrombosis.

The files of 76 patients who were included in the rehabilitation program at our LE unit were reviewed. However, 16 patients were excluded from the study for missing or inconsistent records in their files, and 60 patients (mean age $55.7 \pm 10.3$ years; range 18 to 85 years) were eligible for the analysis. Demographic data including age, marital status, occupation, education, smoking habit, and level of activity and previous medical records including operation date and type, number of chemotherapy cycles, radiotherapy sessions, use of tamoxifen or hormonal therapy, and pain involving upper limb were recruited from the medical files and subsequently analyzed.
The European Organization for Research and Treatment of Cancer Quality of Life- C30 (EORTC QLQC30) for the QoL, the Brief Fatigue Inventory (BFI) for fatigue, the Douleur Neuropathique 4 Questions (DN4) for neuropathic pain, and the Beck Depression Inventory (BDI) for the emotional status were used before and after the treatment.

The EORTC QLQ-C30 questionnaire is a survey conducted to evaluate the QoL in patients with breast cancer, and consists of 30 questions and assesses symptoms that occurred in the previous two weeks. Answers are displayed in a Likert scale: 1 - not at all, 2 - a little, 3 - quite a bit, 4 - very much. The global health scale is composed by two questions asking patients to classify their general health and QoL in the previous week, by rating it from 1 to 7 , in which 1 means poor and 7, excellent. The questionnaires are divided into three scales: global health scale (GHS), functional scale (FS) and symptom scale (SS). ${ }^{[12]}$

Brief Fatigue Inventory, was developed to assess fatigue in cancer patients. This is also used to determine the severity of fatigue occurring during the preceding 24 hours and the interference it causes in the individual's life. It consists of nine questions, which are rated using the Likert scales ranging from 0 to 10 . The mean scores for these nine items are used as global fatigue scores (GFSs); scores of 1-3, 4-6, and 7-10 are categorized as mild, moderate, and severe, respectively. ${ }^{[13]}$

The DN4 questionnaire consists of 10 items. The first seven items are related to pain characteristics and sensations and the remaining three items are related to the examination. For each item, a score of "1" is given if the answer is "Yes" and a score of " 0 " is given if it is "No." The patient is defined to have neuropathic pain, if the sum of all 10 items is calculated to be four or more. ${ }^{[14]}$

The BDI was used to assess the depression levels of the patients. The BDI evaluates 21 symptoms of depression. These symptoms deal with emotions, behavioral changes, and somatic symptoms. Each symptom is rated on a four-point intensity scale. Higher scores indicate more severe depression. The validity and reliability studies of the Turkish version have been previously performed. ${ }^{[15]}$

Lymphedema of the limbs were assessed by a single physiotherapist using the circumferential and volumetric methods before and after the treatment protocol. The circumferential upper limb measurements were carried out with the arm abducted at $30^{\circ}$, starting at the level of the carpometacarpal joint, every $5 \mathrm{~cm}$ 
Table 1. Demographic and clinical characteristics of patients

\begin{tabular}{lccr}
\hline & $\mathrm{n}$ & $\%$ & Mean \pm SD \\
\hline Age (year) & 60 & & $55.7 \pm 10.3$ \\
Gender & & & \\
$\quad$ Male & 2 & 3.3 & \\
$\quad$ Female & 58 & 96.7 & \\
Education level & & & \\
$\quad$ Literate & 1 & 1.7 & \\
$\quad$ Elementary school & 19 & 31.7 & \\
$\quad$ Middle or high school & 37 & 61.6 & \\
$\quad$ University & 3 & 5.0 & $62.5 \pm 52.9$ \\
Postoperative duration (month) & & & $21.1 \pm 40.7$ \\
Duration of lymphedema (month) & & & $8.1 \pm 9.0$ \\
Number of lymph nodes removed & & & $6.4 \pm 3.2$ \\
Number of positive lymph nodes & & & $24.6 \pm 9.9$ \\
Number of chemotherapy cures & & & \\
Number of radiotherapy sessions & & & \\
\hline SD: Standard deviation. & & &
\end{tabular}

proximal to this point along both limbs. Then, a computer program (limb volumes professional version 5.0) was used to convert these values into limb volumes in milliliters.

All patients underwent 20 sessions (1 hour) of CDT for four weeks (5 days per week). This program was comprised of patient education, MLD (self), compression therapy with a short-stretch bandage for $23 \mathrm{~h}$ per day, exercise, and skin care. After four weeks of treatment, Phase II protocol was commenced. This phase included the use of compression clothing to maintain the volume reduction and recommendation of daily exercise, regular application of MLD and skin care. In addition, all patients were advised to lose weight and given detailed brochures about LE and exercise.

A written informed consent was obtained from each patient with a written permission from each treating physician. The study protocol was approved by the institutional local Ethics Committee (Decision number: 16-5/9). The study was conducted in accordance with the principles of the Declaration of Helsinki.

\section{Statistical analysis}

Statistical analysis was performed using the IBM SPSS for Windows version 20.0 (IBM Corp., Armonk, NY, USA). Continuous variables were expressed in mean \pm standard deviation (SD), while categorical variables were expressed in number and percentage. Comparisons of pre- vs post-intervention values for continuous variables were made using the paired t-test. A $p$ value of less than 0.05 was considered statistically significant.

\section{RESULTS}

The study included a total of 60 patients. Demographic and clinical characteristics of all the participants are shown in Table 1.

Table 2. Change in volume of involved limbs and European Organization for Research and Treatment of Cancer Core-30, Beck Depression Inventory, Douleur Neuropathique 4 Question and Brief Fatigue Index scores after treatment

\begin{tabular}{lcccc}
\hline Variable & Before treatment & & After treatment & \\
& Mean \pm SD & & Mean \pm SD & $p$ \\
\hline Affected extremity volume $(\mathrm{mL})$ & $2712.7 \pm 791.2$ & & $2513.6 \pm 678.7$ & $<0.001$ \\
EORTC-Q30-functional & $68.4 \pm 21.0$ & & $73.9 \pm 16.4$ & 0.004 \\
EORTC-Q30-symptom & $20.1 \pm 15.8$ & & $17.0 \pm 12.4$ & 0.066 \\
EORTC-Q30-global health & $58.9 \pm 26.0$ & & $79.4 \pm 20.0$ & $<0.001$ \\
Beck Depression Inventory & $8.4 \pm 9.9$ & & $7.4 \pm 8.8$ & 0.019 \\
Douleur Neuropathique 4 Question & $2.2 \pm 2.4$ & & $1.5 \pm 2.0$ & $<0.001$ \\
Brief Fatigue Index & $3.6 \pm 2.3$ & $3.3 \pm 2.3$ & 0.043 \\
\hline SD: Standard deviation; EORTC-Q30: European Organization for Research and Treatment of Cancer Core-30. &
\end{tabular}


For the evaluation of the treatment effectiveness, the mean volumes of the limbs before and after treatment protocol were analyzed. Accordingly, these values were $2,712.7 \pm 791.2 \mathrm{~mL}$ and $2,513.6 \pm 678.7$ $\mathrm{mL}$, respectively, indicating a statistically significant reduction in the volume of the involved limbs after the treatment $(\mathrm{p}<0.001)($ Table 2$)$.

In addition, there was a significant improvement in the general health status and functional scores of the EORTC QLQ-C30 ( $<0.001$ and $\mathrm{p}=0.004$, respectively) and a non-significant reduction in the symptom scale scores $(\mathrm{p}=0.066)$ (Table 2$)$.

The baseline DN4 score was calculated to be $2.2 \pm 2.4$, and it was improved to $1.5 \pm 2.0$ with treatment (CDT) $(\mathrm{p}<0.001)$. In addition, the BFI and BDI scores of the patients were significantly improved after the treatment ( $\mathrm{p}=0.043$ and $\mathrm{p}=0.019)$ (Table 2).

We found no adverse effects related to the treatment, and no additional drugs were used for LE during the study.

\section{DISCUSSION}

In this study, we investigated the effects of CDT in the BCRL patients, and we found that it was successful for reducing arm volume, improving QoL or mood, and decreasing level of fatigue or neuropathic pain. Complete decongestive therapy was well-tolerated by the patients and no adverse effect or worsening in LE was reported.

Major concerns in the management of LE include preventing the disease progression, reducing or preserving the size of the limb (to the fullest possible extent), reducing complication rates, and relieving symptoms related to LE. Most important of these can be defined as QoL. Quality of life has been used often as the outcome variable in the study of cancer and its associated problems. More importantly, poorer QoL outcomes in patients with BCRL resulting in decreased physical functioning, and psychological and social well-being were recorded in previous studies. ${ }^{[16]}$ Ahmed et al. ${ }^{[17]}$ reviewed the database of Iowa Women's Health Study and reported that the women with selfreported LE ( $8.1 \%$ of the sample) and the women with arm symptoms (37.2\% of the sample) had lower healthrelated quality of life (HRQoL) scores, compared to those without LE or arm symptoms.

In addition, CDT has been accepted to be of the most effective treatment protocols for LE. A recent study by Noh et al. ${ }^{[18]}$ which investigated the changing patterns of edema and QoL after CDT included 35 patients with upper limb LE who were applied CDT for two weeks before self-administered home therapy program. After evaluating the patients with Short Form-36 (SF-36; Korean version), they reported significant improvement in both QoL and LE volumes. A systematic review including 26 studies about CDT between 2004 and 2011 suggested results supporting the view that CDT decreases limb volumes and improves QoL. ${ }^{[4]}$ Reduction of limb volume in LE patients induces better physical functionality, decreases fear of movement, and subsequently improves QoL. In addition to the existing literature, our results also suggest an improvement in all EORTC scores after the treatment; and thus, we believe that CDT, which seems to improve QoL, should be considered as the first-line treatment protocol in LE patients.

Cancer-related fatigue $(\mathrm{CRF})$ is defined as a distressing, persistent, subjective sense of physical, emotional, and/or cognitive tiredness that has an adverse impact on daily activities and relates to cancer or its treatment. ${ }^{[19]}$ Currently, the pathophysiology of CRF remains a mystery, and it can be present during the treatment or even after many years. Although CRF is common and seriously impairs QoL, in general practice, it is usually an underestimated issue among physicians. Very little is known about CRF in patients with BCRL; however, breast cancer survivors with LE in the ipsilateral upper extremity usually report experience of fatigue as well as swelling, firmness, tightness or heaviness. ${ }^{[20]}$ Perhaps, these symptoms may be the earliest indicator of increasing interstitial pressure changes associated with LE. Review of the literature revealed a single study investigating the outcomes of LE treatment in patients with CRF. Gurdal et al. ${ }^{[21]}$ compared two different methods in LE treatment, and suggested improvements in fatigue scores of the patients. In our study, we also investigated the effect of CDT on reducing CRF and determined improvements in fatigue scores of patients after the treatment. We believe that the improvement in fatigue scores after CDT can be attributed to volume reduction of limbs and eventual loss of sense of heavy limbs in patients, which, in turn, can induce better physical functionality and improved QoL. Another point of view is that CDT can also indirectly reduce fatigue by improving both patients' mental health and QoL.

Until now, the psychological sequelae of treatment for breast cancer have been well-documented. Lymphedema, which may undoubtedly be an unpleasant complication of breast cancer, can cause physical and functional instability along with low self-esteem or negative mood (i.e., distressed, anxious, 
fierce or melancholic mood), and these can result in eventual clinical depression or anxiety in patients. ${ }^{[22]}$ All these factors aggravate symptoms in upper limbs and impair QoL simultaneously, while causing social isolation or non-compliance with treatment. Additionally, neglection of psychological status and sequelae in these patients can result in inadequate and more expensive medical care. ${ }^{[5]}$ In this regard, apart from a precise diagnosis, considering of an effective treatment strategy for this type of depressive mood in patients with BRCL seems to be essential. We found only one study investigating the effects of CDT in LE from the perspective of psychology. A recent study by Atalay et al. ${ }^{[23]}$ included 58 patients with BCRL to investigate the impact of Phase 1 CDT on the level of clinical depression. Their results suggest a significant reduction in the BDI score and a significant positive correlation between depression levels and circumference measurement. This study, along with the literature, determines psychological sequelae of arm morbidity with BDI. Our results suggest significant improvement in depression scores accompanied by reduction in LE. This improvement in the group may be explained by decreasing the volume of limbs, while improving the QoL; thus, patients have more pain relief and less cosmetic problems.

Another important issue in these patients is pain. A discomforting sense of pain that involves extremities of patients with BCRL may be the early indicator of increasing interstitial pressure associated with LE. ${ }^{[24,25]}$ This complication has been reported in 20 to $50 \%$ of BCRL patients. Pain is often described by patients as burning, aching, constriction, scar sensitivity, discomfort, or tenderness. Undoubtedly, pain limits daily activities to some degree. ${ }^{[26]}$ Some of the factors contributing to pain may be noted as mastectomy, axillary lymph node dissection, trauma to the tissues during the surgery, dissection of the intercostobrachial nerve, or intraoperative injury of axillary nerve branches.

There are various reports suggesting that pain is associated with poor QoL. Some authors have advocated that complete decongestive therapy significantly decrease girth together with Visual Analog Scale scores for pain. ${ }^{[27,28]}$ Therefore, informing patients prior to any surgical intervention seems to essential and beneficial. Consistent with previous literature, we also showed a significant reduction in the DN4 scores after CDT; certainly, this may be attributed to both the reduction of limb volumes and a better mood of the patients with improved QoL.
Of note, this study is the first to undertake a detailed analysis of CDT in the management of LE and related symptoms such as depression, fatigue and neuropathic pain. In addition, it has a large sample size and CDT was applied to the patients by a single physiotherapist to have a standard approach. However, the limitations of the study are lack of benefit-cost analysis, long-term follow-up results, and a detailed analysis regarding the neuropathic pain.

In conclusion, BCRL is a serious complication which may lead to symptoms such as pain, fatigue, depression, and eventual deterioration in the QoL. Certainly, CDT is an effective and safe method to achieve not only a significant volume reduction in limbs involved by LE, but also favorable outcomes in the management of the aforementioned issues. It is essential to apply an immediate management protocol for BCRL as well as to encourage serious efforts by both government, other organizations, and initiatives to increase public awareness.

\section{Declaration of conflicting interests}

The authors declared no conflicts of interest with respect to the authorship and/or publication of this article.

\section{Funding}

The authors received no financial support for the research and/or authorship of this article.

\section{REFERENCES}

1. Ferlay J, Soerjomataram I, Ervik M, Dikshit R, Eser S, Mathers C, et al. GLOBOCAN 2012: Estimated Cancer Incidence, Mortality and Prevalence Worldwide in 2012. Lyon: International Agency for Research on Cancer; 2014. Available from: http://globocan.iarc.fr [Accessed: January 16, 2015]

2. Eyigor S, Kanyilmaz S. Exercise in patients coping with breast cancer: An overview. World J Clin Oncol 2014;5:406-11.

3. Salonen P, Rantanen A, Kellokumpu-Lehtinen PL, Huhtala $\mathrm{H}$, Kaunonen $\mathrm{M}$. The quality of life and social support in significant others of patients with breast cancer--a longitudinal study. Eur J Cancer Care (Engl) 2014;23:274-83.

4. Lasinski BB, McKillip Thrift K, Squire D, Austin MK, Smith $\mathrm{KM}$, Wanchai A, et al. A systematic review of the evidence for complete decongestive therapy in the treatment of lymphedema from 2004 to 2011. PM R 2012;4:580-601.

5. McWayne J, Heiney SP. Psychologic and social sequelae of secondary lymphedema: a review. Cancer 2005;104:457-66.

6. Smoot B, Wong J, Cooper B, Wanek L, Topp K, Byl N, et al. Upper extremity impairments in women with or without lymphedema following breast cancer treatment. J Cancer Surviv 2010;4:167-78.

7. Schmitz KH, Ahmed RL, Troxel AB, Cheville A, LewisGrant L, Smith R, et al. Weight lifting for women at risk for breast cancer-related lymphedema: a randomized trial. JAMA 2010;304:2699-705. 
8. Pinto M, Gimigliano F, Tatangelo F, Megna M, Izzo F, Gimigliano R, et al. Upper limb function and quality of life in breast cancer related lymphedema: a cross-sectional study. Eur J Phys Rehabil Med 2013;49:665-73.

9. Shih YC, Xu Y, Cormier JN, Giordano S, Ridner SH, Buchholz TA, et al. Incidence, treatment costs, and complications of lymphedema after breast cancer among women of working age: a 2-year follow-up study. J Clin Oncol 2009;27:2007-14.

10. Mayrovitz HN. The standard of care for lymphedema: current concepts and physiological considerations. Lymphat Res Biol 2009;7:101-8.

11. Forner-Cordero I, Muñoz-Langa J, Forner-Cordero A, DeMiguel-Jimeno JM. Predictive factors of response to decongestive therapy in patients with breast-cancer-related lymphedema. Ann Surg Oncol 2010;17:744-51.

12. Aaronson NK, Ahmedzai S, Bergman B, Bullinger M, Cull A, Duez NJ, et al. The European Organization for Research and Treatment of Cancer QLQ-C30: a qualityof-life instrument for use in international clinical trials in oncology. J Natl Cancer Inst 1993;85:365-76.

13. Mendoza TR, Wang XS, Cleeland CS, Morrissey M, Johnson $\mathrm{BA}$, Wendt JK, et al. The rapid assessment of fatigue severity in cancer patients: use of the Brief Fatigue Inventory. Cancer 1999;85:1186-96.

14. Bouhassira D, Attal N, Alchaar H, Boureau F, Brochet B, Bruxelle J, et al. Comparison of pain syndromes associated with nervous or somatic lesions and development of a new neuropathic pain diagnostic questionnaire (DN4). Pain 2005;114:29-36.

15. Beck AT, Ward CH, Mendelson M, Mock J, Erbaugh J. An inventory for measuring depression. Arch Gen Psychiatry 1961;4:561-71.

16. Taghian NR, Miller CL, Jammallo LS, O'Toole J, Skolny MN. Lymphedema following breast cancer treatment and impact on quality of life: a review. Crit Rev Oncol Hematol 2014;92:227-34.

17. Ahmed RL, Prizment A, Lazovich D, Schmitz KH, Folsom AR. Lymphedema and quality of life in breast cancer survivors: the Iowa Women's Health Study. J Clin Oncol 2008;26:5689-96.
18. Noh S, Hwang JH, Yoon TH, Chang HJ, Chu IH, Kim JH. Limb Differences in the Therapeutic Effects of Complex Decongestive Therapy on Edema, Quality of Life, and Satisfaction in Lymphedema Patients. Ann Rehabil Med 2015;39:347-59.

19. Mock V, Atkinson A, Barsevick A, Cella D, Cimprich B, Cleeland C, et al. NCCN Practice Guidelines for CancerRelated Fatigue. Oncology (Williston Park) 2000;14:151-61.

20. Fu MR, Rosedale M. Breast cancer survivors' experiences of lymphedema-related symptoms. J Pain Symptom Manage 2009;38:849-59.

21. Gurdal SO, Kostanoglu A, Cavdar I, Ozbas A, Cabioglu N, Ozcinar B, et al. Comparison of intermittent pneumatic compression with manual lymphatic drainage for treatment of breast cancer-related lymphedema. Lymphat Res Biol 2012;10:129-35.

22. Alcorso J, Sherman KA. Factors associated with psychological distress in women with breast cancer-related lymphoedema. Psychooncology 2016;25:865-72.

23. Atalay OT, Özkir A, Çalik BB, Baskan E, Taşkin H. Effects of phase I complex decongestive physiotherapy on physical functions and depression levels in breast cancer related lymph edema. J Phys Ther Sci 2015;27:865-70.

24. Fu MR, Axelrod D, Cleland CM. Symptom report in detecting breast cancer-related lymphedema. Breast Cancer (Dove Med Press) 2015;7:345-52.

25. Fu MR. Breast cancer-related lymphedema: Symptoms, diagnosis, risk reduction, and management. World J Clin Oncol 2014;5:241-7.

26. Hidding JT, Beurskens $\mathrm{CH}$, van der Wees PJ, van Laarhoven HW, Nijhuis-van der Sanden MW. Treatment related impairments in arm and shoulder in patients with breast cancer: a systematic review. PLoS One 2014;9:e96748.

27. Moseley AL, Carati CJ, Piller NB. A systematic review of common conservative therapies for arm lymphoedema secondary to breast cancer treatment. Ann Oncol 2007;18:639-46.

28. Hamner JB, Fleming MD. Lymphedema therapy reduces the volume of edema and pain in patients with breast cancer. Ann Surg Oncol 2007;14:1904-8. 